\title{
Effects of boundary conditions on the parametric resonance of cylindrical shells under axial loading
}

\author{
T.Y. Ng and K.Y. Lam \\ Centre for Computational Mechanics, Department of \\ Mechanical \& Production Engineering, National \\ University of Singapore, 10 Kent Ridge Crescent, \\ Singapore 119260
}

Received 9 January 1998

Revised 6 November 1998

\begin{abstract}
In this paper, a formulation for the dynamic stability analysis of circular cylindrical shells under axial compression with various boundary conditions is presented. The present study uses Love's first approximation theory for thin shells and the characteristic beam functions as approximate axial modal functions. Applying the Ritz procedure to the Lagrangian energy expression yields a system of Mathieu-Hill equations the stability of which is analyzed using Bolotin's method. The present study examines the effects of different boundary conditions on the parametric response of homogeneous isotropic cylindrical shells for various transverse modes and length parameters.
\end{abstract}

\section{Introduction}

The dynamic stability of cylindrical shells has received much attention over the years. Yao [16] was the first to investigate dynamic stability in cylindrical shells and the loading used in this work considered both radial and axial directions. Shirakawa [14] considered in-plane inertia and in-plane disturbances in a study of a similar problem. Argento and Scott [1,2] and Argento [3] in a series of papers dealt with this problem for a composite shell subjected to periodic axial and torsional loading. The boundary conditions considered were for a shell clamped at both ends. Bert and Birman [4], in the parametric instability study of thick orthotropic cylindrical shells considered simplysupported end conditions. Koval [7], in the study of the effects of longitudinal resonance on the parametric stability of an axially excited cylindrical shell considered cases with simply-supported end conditions. Liao and Cheng $[9,10]$, using the finite element method for the dynamic stability of stiffened laminated composite shells subjected to in-plane pulsating forces, considered shells that were clamped at the boundaries. Radwan and Genin [13], in the dynamic instability of cylindrical shells, considered simply-supported cases. In the dynamic instability of truncated conical shells, Massalas et al. [11] considered only clamped cases. Nagai and Yamaki [12], in the dynamic stability analysis of cylindrical shells under periodic compressive forces, compared results for simply-supported and clamped edge conditions.

A literature search showed that a study on the effects of boundary conditions on the dynamic stability of circular cylindrical shells, with the inclusion of free edge conditions into the comparisons, is not available. Further, to the authors' knowledge, no results are available for the dynamic stability of shells with free end conditions. A comprehensive study as such would be interesting as it would shed light on the effects of boundary conditions on the instability regions.

\section{Theory and formulation}

The cylindrical shell with constant thickness $h$, radius $R$ and length $L$ is in the coordinate system as shown in Fig. 1. The periodic extensional axial load per unit length is given by

$$
N_{\mathrm{a}}(x, t)=N_{\mathrm{o}}+N_{\mathrm{s}} \cos P t
$$

where $P$ is the frequency of excitation in radians per unit time.

The deformations of the shell are defined as the displacements $u, v$ and $w$ in the axial, circumferential and radial directions respectively. 


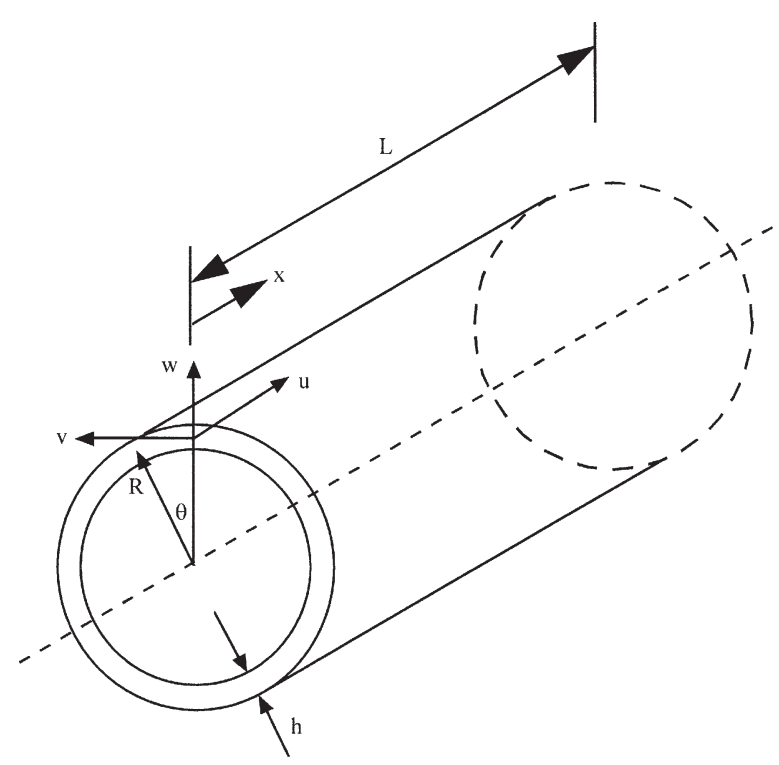

Fig. 1. Coordinate system of the cylindrical shell.

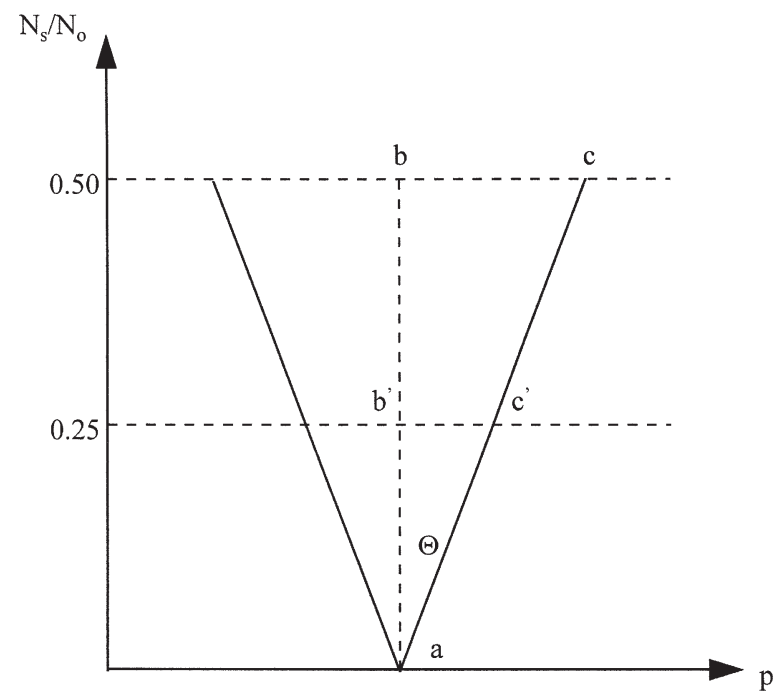

Fig. 2. An unstable region in the $N_{\mathrm{s}} / N_{\mathrm{o}}-p$ plane.

Using Love's first approximation theory, the axial, circumferential and in-surface shear strains, $e_{x}, e_{\theta}$ and $e_{x \theta}$ are defined as

$$
\begin{gathered}
e_{x}=e_{1}-z k_{1}, \\
e_{\theta}=e_{2}-z k_{2}, \\
e_{x \theta}=\gamma-2 z \tau,
\end{gathered}
$$

where

$$
\begin{aligned}
& e_{1}=\frac{\partial u}{\partial x}, \quad e_{2}=\frac{1}{R}\left(\frac{\partial v}{\partial \theta}+w\right), \\
& \gamma=\frac{\partial v}{\partial x}+\frac{1}{R} \frac{\partial u}{\partial \theta}
\end{aligned}
$$

and

$$
\begin{aligned}
& k_{1}=\frac{\partial^{2} w}{\partial x^{2}}, \quad k_{2}=\frac{1}{R^{2}}\left(\frac{\partial^{2} w}{\partial \theta^{2}}-\frac{\partial v}{\partial \theta}\right), \\
& \tau=\frac{1}{R}\left(\frac{\partial^{2} w}{\partial x \partial \theta^{2}}-\frac{1}{2} \frac{\partial v}{\partial x}\right) .
\end{aligned}
$$

The middle surface strains are $e_{1}, e_{2}$ and $\gamma$ and the middle surface curvatures are $k_{1}, k_{2}$ and $2 \tau$. Assuming plane stress, a thin shell follows the two-dimensional Hooke's law

$$
\{\sigma\}=[Q]\{e\},
$$

where

$$
\begin{aligned}
& \{\sigma\}^{\mathrm{T}}=\left\{\sigma_{x} \sigma_{\theta} \sigma_{x \theta}\right\}, \\
& {[Q]=\left[\begin{array}{ccc}
\frac{E}{1-\nu^{2}} & \frac{\nu E}{1-\nu^{2}} & 0 \\
\frac{\nu E}{1-\nu^{2}} & \frac{E}{1-\nu^{2}} & 0 \\
0 & 0 & G_{12}
\end{array}\right] \text {, }} \\
& \{e\}^{\mathrm{T}}=\left\{e_{x} e_{\theta} e_{x \theta}\right\},
\end{aligned}
$$

where $E$ is the elastic modulus and $\nu$ is the Poisson's ratio. The shear modulus is given as

$$
G_{12}=\frac{E}{2(1+\nu)} .
$$

The force and moment resultants are defined as

$$
\begin{gathered}
\left(N_{x}, N_{\theta}, N_{x \theta}\right)=\int_{-h / 2}^{h / 2}\left(\sigma_{x}, \sigma_{\theta}, \sigma_{x \theta}\right) \mathrm{d} z \\
\left(M_{x}, M_{\theta}, M_{x \theta}\right)=\int_{-h / 2}^{h / 2}\left(\sigma_{x}, \sigma_{\theta}, \sigma_{x \theta}\right) z \mathrm{~d} z .
\end{gathered}
$$

Simplifying, one obtains the following constitutive equation

$$
\{N\}=[S]\{\varepsilon\},
$$

where

$$
\{N\}^{\mathrm{T}}=\left\{N_{x} N_{\theta} N_{x \theta} M_{x} M_{\theta} M_{x \theta}\right\},
$$


$[S]=\left[\begin{array}{cccccc}A_{11} & A_{12} & 0 & 0 & 0 & 0 \\ A_{12} & A_{22} & 0 & 0 & 0 & 0 \\ 0 & 0 & A_{66} & 0 & 0 & 0 \\ 0 & 0 & 0 & D_{11} & D_{12} & 0 \\ 0 & 0 & 0 & D_{12} & D_{22} & 0 \\ 0 & 0 & 0 & 0 & 0 & D_{66}\end{array}\right]$,

$\{\varepsilon\}^{\mathrm{T}}=\left\{e_{1} e_{2} \gamma k_{1} k_{2} 2 \tau\right\}$.

The extensional stiffnesses $A_{i j}$ 's and bending stiffnesses $D_{i j}$ 's are defined as

$$
\left\{A_{i j}, D_{i j}\right\}=\int_{-h / 2}^{h / 2} Q_{i j}\left\{1, z^{2}\right\} \mathrm{d} z
$$

The total strain energy $U_{\varepsilon}$ and total kinetic energy $T$ of the cylindrical shell can be written as

$$
\begin{aligned}
U_{\varepsilon}= & \frac{1}{2} \int_{0}^{L} \int_{0}^{2 \pi} \varepsilon^{\mathrm{T}}[S] \varepsilon R \mathrm{~d} \theta \mathrm{d} x, \\
T= & \frac{1}{2} \int_{0}^{L} \int_{0}^{2 \pi} \rho_{t}\left[\left(\frac{\partial u}{\partial t}\right)^{2}+\left(\frac{\partial v}{\partial t}\right)^{2}\right. \\
& \left.+\left(\frac{\partial w}{\partial t}\right)^{2}\right] R \mathrm{~d} \theta \mathrm{d} x,
\end{aligned}
$$

where $\rho_{t}$ is the mass per unit surface area ( $\rho$ being the mass density) defined as

$$
\rho_{t}=\int_{-h / 2}^{h / 2} \rho \mathrm{d} z
$$

The strain energy $U_{\mathrm{a}}$ due to the axial loading can be written as

$U_{\mathrm{a}}=\frac{1}{2} \int_{0}^{L} \int_{0}^{2 \pi} N_{\mathrm{a}}\left[\left(\frac{\partial v}{\partial x}\right)^{2}+\left(\frac{\partial w}{\partial x}\right)^{2}\right] R \mathrm{~d} \theta \mathrm{d} x$

The displacement field for a cylindrical shell of arbitrary boundary conditions can be expressed in general form as given in Blevins [5]

$$
\begin{aligned}
u & =A \frac{\partial \phi(x)}{\partial x} \cos (n \theta) q(t), \\
v & =B \phi(x) \sin (n \theta) q(t), \\
w & =C \phi(x) \cos (n \theta) q(t),
\end{aligned}
$$

where $A, B$ and $C$ are constants and $\phi(x)$ is the axial modal function satisfying the required boundary conditions at both ends of the shell. The circumferential wave number is $n$ and $q(t)$ is a generalized coordinate.

Defining the Lagrangian function

$$
L=T-U_{\varepsilon}-U_{\mathrm{a}}
$$

and applying the Ritz procedure with

$$
\frac{\partial L}{\partial A}=0, \quad \frac{\partial L}{\partial B}=0, \quad \frac{\partial L}{\partial C}=0
$$

one obtains, after rearranging, a system of MathieuHill equations given by

$$
\mathbf{M} \ddot{\mathbf{q}}+(\mathbf{K}-\cos P t \mathbf{Q}) \mathbf{q}=0,
$$

where $\mathbf{M}, \mathbf{K}$ and $\mathbf{Q}$ are $3 \times 3$ matrices and $\ddot{\mathbf{q}}$ and $\mathbf{q}$ are column vectors consisting of the $\dddot{q}$ 's and $q$ 's (corresponding to the axial, circumferential and transverse modes) respectively.

\section{Stability analysis}

Equation (26) is in the form of a second order differential equation with periodic coefficients of the Mathieu-Hill type. The regions of unstable solutions are separated by periodic solutions having period $T$ and $2 T$ with $T=2 \pi / P$. The solutions with period $2 T$ are of greater practical importance as the widths of these unstable regions are usually larger than those associated with solutions having period $T$. Using Bolotin's [6] approach, as a first approximation, the periodic solutions with period $2 T$ can be sought from the following form

$$
\mathbf{f}=\mathbf{a} \sin \frac{P t}{2}+\mathbf{b} \cos \frac{P t}{2},
$$

where $\mathbf{a}$ and $\mathbf{b}$ are arbitrary vectors.

Substituting Eq. (27) into Eq. (26) and equating the coefficients of the $\sin (P t / 2)$ and $\cos (P t / 2)$ terms, a set of linear homogeneous algebraic equations in terms of $\mathbf{a}$ and $\mathbf{b}$ can be obtained. The conditions for non-trivial solutions are given by

$$
\operatorname{det}\left[\begin{array}{cc}
A_{I J} & 0 \\
0 & B_{I J}
\end{array}\right]=0,
$$

where 

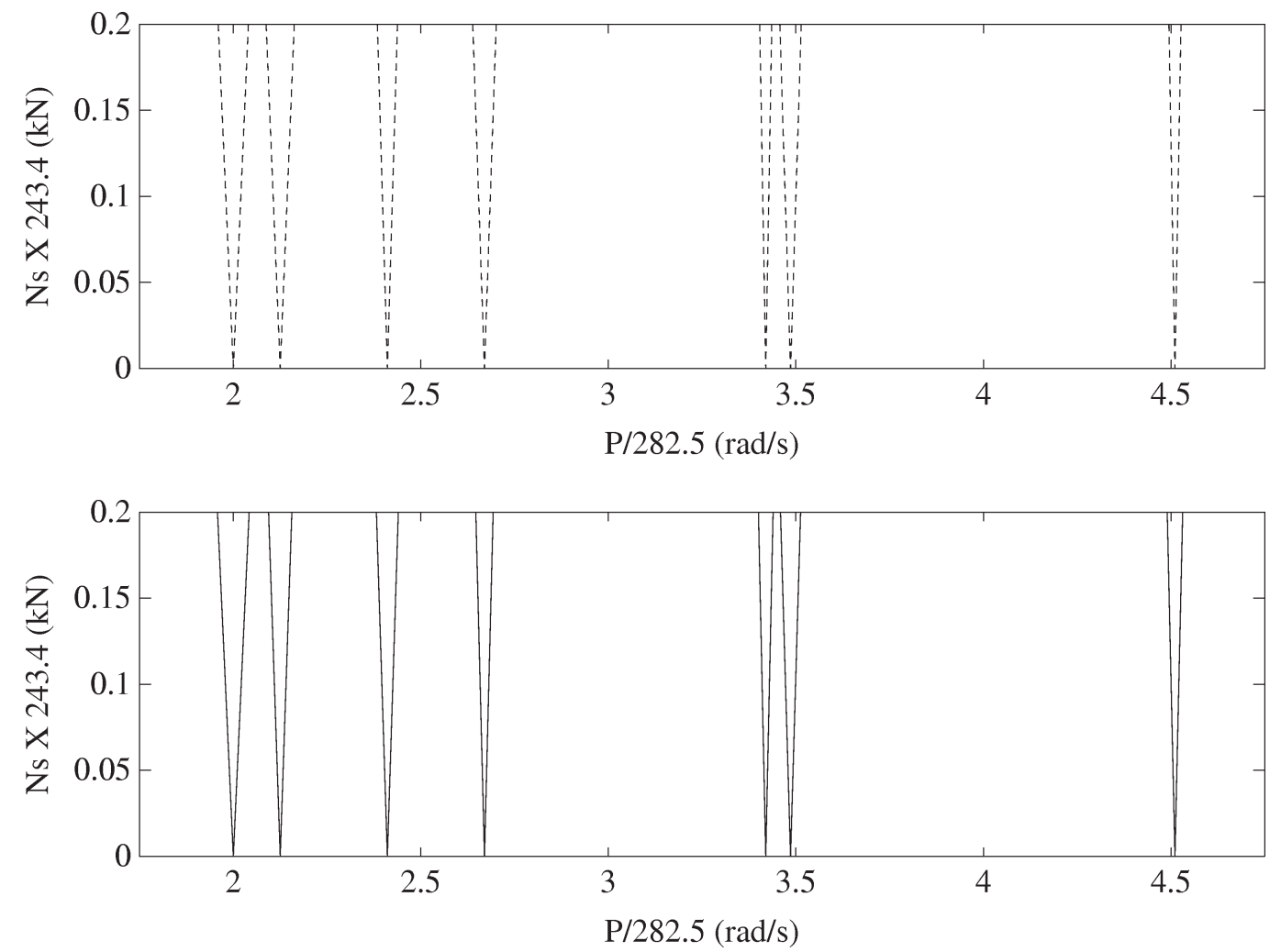

Fig. 3. Comparison of results for the dynamic stability of analysis of a composite laminated cylindrical shell of lamination scheme [ $90^{\circ} / 0^{\circ} / 90^{\circ}$ ] and physical parameters as described in Argento and Scott [3]. $N_{\mathrm{o}}=0.3 \times 243.4 \mathrm{kN} . m=1, n=4,5,3,6,2,7,8$ (from left to right). '- - ', Argento and Scott [3], '_-, Present.

$$
\begin{aligned}
& A_{I J}=\mathbf{K}_{I J}-\frac{1}{2} \mathbf{Q}_{I J}-\frac{1}{4} P^{2} \mathbf{M}_{I J}, \\
& B_{I J}=\mathbf{K}_{I J}+\frac{1}{2} \mathbf{Q}_{I J}-\frac{1}{4} P^{2} \mathbf{M}_{I J} .
\end{aligned}
$$

Instead of solving the above nonlinear geometric equations for $P$, the above equation can be rearranged to the standard form of a generalized eigenvalue problem

$$
\begin{gathered}
\operatorname{det}\left[\left(\begin{array}{cc}
\mathbf{K}_{I J}-\frac{1}{2} \mathbf{Q}_{I J} & \mathbf{0} \\
\mathbf{0} & \mathbf{K}_{I J}+\frac{1}{2} \mathbf{Q}_{I J}
\end{array}\right)\right. \\
\left.-P^{2}\left(\begin{array}{cc}
\frac{1}{4} \mathbf{M}_{I J} & \mathbf{0} \\
\mathbf{0} & \frac{1}{4} \mathbf{M}_{I J}
\end{array}\right)\right]=0
\end{gathered}
$$

where $\mathbf{0}$ is a $3 \times 3$ null matrix.

\section{Axial modal functions and boundary conditions}

The axial modal function $\phi(x)$ is chosen to satisfy the required end conditions at the two ends of the cylindrical shell. In this study, the characteristic or analytic beam functions have been chosen. First we define the boundary conditions. The modal function defines the transverse displacement, the rotation corresponds to its first derivative with respect to $x$, the bending moment corresponds to its second derivative and the transverse shear force corresponds to its third derivative.

At a clamped boundary, transverse displacement and rotation are prohibited,

$$
\phi=\frac{\partial \phi}{\partial x}=0
$$

At a simply-supported boundary, transverse displacement and bending moment are prohibited,

$$
\phi=\frac{\partial^{2} \phi}{\partial x^{2}}=0
$$

At a free boundary, bending moment and transverse shear force are prohibited,

$$
\frac{\partial^{2} \phi}{\partial x^{2}}=\frac{\partial^{3} \phi}{\partial x^{3}}=0 .
$$


Table 1

Unstable regions for a cylindrical shell of length ratios $L / R=1,3,5$, tensile loading of $\eta_{\mathrm{o}}=$ $0.5 \eta_{\mathrm{cr}}$ and with axial wave number $m=1$

\begin{tabular}{|c|c|c|c|c|c|}
\hline & & SS-SS & $\mathrm{C}-\mathrm{C}$ & F-F & \\
\hline \multirow[t]{6}{*}{$L / R=1$} & $n=1$ & Pt. of origin $p$ & 1.7226704 & 1.7996997 & 2.0682982 \\
\hline & & Angle subtended $\Theta \times 10^{-2}$ & 1.5704949 & 1.8768033 & 6.5162280 \\
\hline & $n=2$ & Pt. of origin $p$ & 1.3471428 & 1.4512196 & 1.9467126 \\
\hline & & Angle subtended $\Theta \times 10^{-2}$ & 2.0082898 & 2.3245120 & 6.9255557 \\
\hline & $n=3$ & Pt. of origin $p$ & 1.0214074 & 1.1677581 & 1.7514490 \\
\hline & & Angle subtended $\Theta \times 10^{-2}$ & 2.6347333 & 2.8818143 & 7.6562853 \\
\hline \multirow[t]{6}{*}{$L / R=3$} & $n=1$ & Pt. of origin $p$ & 0.7532045 & 0.9077726 & 1.4230583 \\
\hline & & Angle subtended $\Theta \times 10^{-2}$ & 0.3875676 & 0.4133224 & 0.9388126 \\
\hline & $n=2$ & Pt. of origin $p$ & 0.3797018 & 0.5469822 & 0.8110883 \\
\hline & & Angle subtended $\Theta \times 10^{-2}$ & 0.7766855 & 0.6837076 & 1.7209182 \\
\hline & $n=3$ & Pt. of origin $p$ & 0.2294124 & 0.3604280 & 0.5074538 \\
\hline & & Angle subtended $\Theta \times 10^{-2}$ & 1.2886346 & 1.0342319 & 2.8467950 \\
\hline \multirow[t]{6}{*}{$L / R=5$} & $n=1$ & Pt. of origin $p$ & 0.3782150 & 0.5317407 & 0.7731918 \\
\hline & & Angle subtended $\Theta \times 10^{-2}$ & 0.2728141 & 0.2526790 & 0.5411772 \\
\hline & $n=2$ & Pt. of origin $p$ & 0.1661971 & 0.2817677 & 0.3716542 \\
\hline & & Angle subtended $\Theta \times 10^{-2}$ & 0.6396071 & 0.4763392 & 1.3680922 \\
\hline & $n=3$ & Pt. of origin $p$ & 0.1103028 & 0.1757311 & 0.2341207 \\
\hline & & Angle subtended $\Theta \times 10^{-2}$ & 0.9610056 & 0.7609491 & 2.2336453 \\
\hline
\end{tabular}

Table 2

Unstable regions for a cylindrical shell of length ratios $L / R=10,20,30$, tensile loading of $\eta_{\mathrm{o}}=0.5 \eta_{\mathrm{cr}}$ and with axial wave number $m=1$

\begin{tabular}{|c|c|c|c|c|c|}
\hline & & SS-SS & $\mathrm{C}-\mathrm{C}$ & F-F & \\
\hline \multirow[t]{6}{*}{$L / R=10$} & $n=6$ & Pt. of origin $p$ & 0.2026213 & 0.2036963 & 0.2148305 \\
\hline & & Angle subtended $\Theta \times 10^{-3}$ & 1.3389081 & 1.6595008 & 6.2934898 \\
\hline & $n=7$ & Pt. of origin $p$ & 0.2768784 & 0.2776116 & 0.2862707 \\
\hline & & Angle subtended $\Theta \times 10^{-3}$ & 0.9806043 & 1.2188422 & 4.7387204 \\
\hline & $n=8$ & Pt. of origin $p$ & 0.3629780 & 0.3635353 & 0.3705403 \\
\hline & & Angle subtended $\Theta \times 10^{-3}$ & 0.7482907 & 0.9312051 & 3.6674125 \\
\hline \multirow[t]{6}{*}{$L / R=20$} & $n=6$ & Pt. of origin $p$ & 0.2001379 & 0.2003568 & 0.2032419 \\
\hline & & Angle subtended $\Theta \times 10^{-3}$ & 0.3393152 & 0.4224544 & 1.6723056 \\
\hline & $n=7$ & Pt. of origin $p$ & 0.2749706 & 0.2751334 & 0.2773427 \\
\hline & & Angle subtended $\Theta \times 10^{-3}$ & 0.2470225 & 0.3077178 & 1.2267132 \\
\hline & $n=8$ & Pt. of origin $p$ & 0.3614304 & 0.3615603 & 0.3633337 \\
\hline & & Angle subtended $\Theta \times 10^{-3}$ & 0.1879495 & 0.2341900 & 0.9368355 \\
\hline \multirow[t]{6}{*}{$L / R=30$} & $n=6$ & Pt. of origin $p$ & 0.1996829 & 0.1997758 & 0.2010669 \\
\hline & & Angle subtended $\Theta \times 10^{-3}$ & 0.1511874 & 0.1883608 & 0.7521679 \\
\hline & $n=7$ & Pt. of origin $p$ & 0.2746192 & 0.2746898 & 0.2756755 \\
\hline & & Angle subtended $\Theta \times 10^{-3}$ & 0.1099426 & 0.1370065 & 0.5488522 \\
\hline & $n=8$ & Pt. of origin $p$ & 0.3611445 & 0.3612015 & 0.3619916 \\
\hline & & Angle subtended $\Theta \times 10^{-3}$ & 0.0836055 & 0.1041976 & 0.4180708 \\
\hline
\end{tabular}

Thus for clamped ends,

$$
\begin{aligned}
\phi= & C_{1}(\cos \varphi x-\cosh \varphi x) \\
& +C_{2}(\sin \varphi x-\sinh \varphi x),
\end{aligned}
$$

where

$$
\begin{aligned}
& C_{1}(\cos \varphi L-\cosh \varphi L) \\
& \quad+C_{2}(\sin \varphi L-\sinh \varphi L)=0,
\end{aligned}
$$



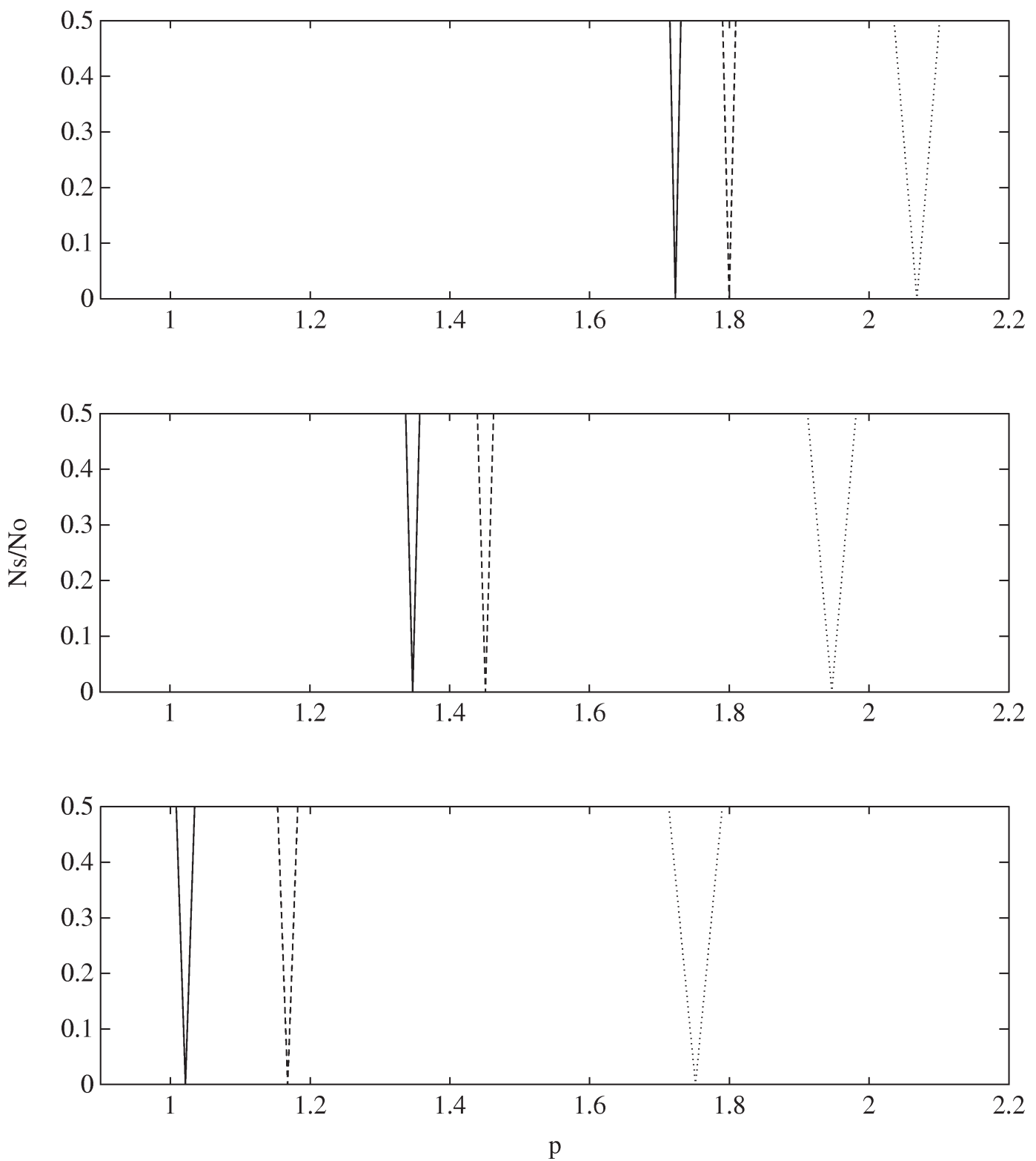

Fig. 4. Unstable regions for a cylindrical shell of length ratio $L / R=1$, tensile loading of $\eta_{\mathrm{o}}=0.5 \eta_{\mathrm{cr}}$ and with axial wave number $m=1$. '_-', SS-SS, '- - -', C-C, ' ' . . ', F-F. Upper diagram $-n=1$. Middle diagram $-n=2$. Lower diagram $-n=3$.

$$
\begin{aligned}
& C_{1}(\sin \varphi L+\sinh \varphi L) \\
& \quad+C_{2}(-\cos \varphi L+\cosh \varphi L)=0
\end{aligned}
$$

with the frequency equation being

$$
\cos \varphi L \cosh \varphi L=1 .
$$

For simply-supported ends,

$$
\phi=C_{1} \sin \varphi x,
$$

where

$$
\varphi=\frac{m \pi}{L} .
$$

For free ends,

$$
\begin{aligned}
\phi= & C_{1}(\cos \varphi x+\cosh \varphi x) \\
& +C_{2}(\sin \varphi x+\sinh \varphi x),
\end{aligned}
$$

where 

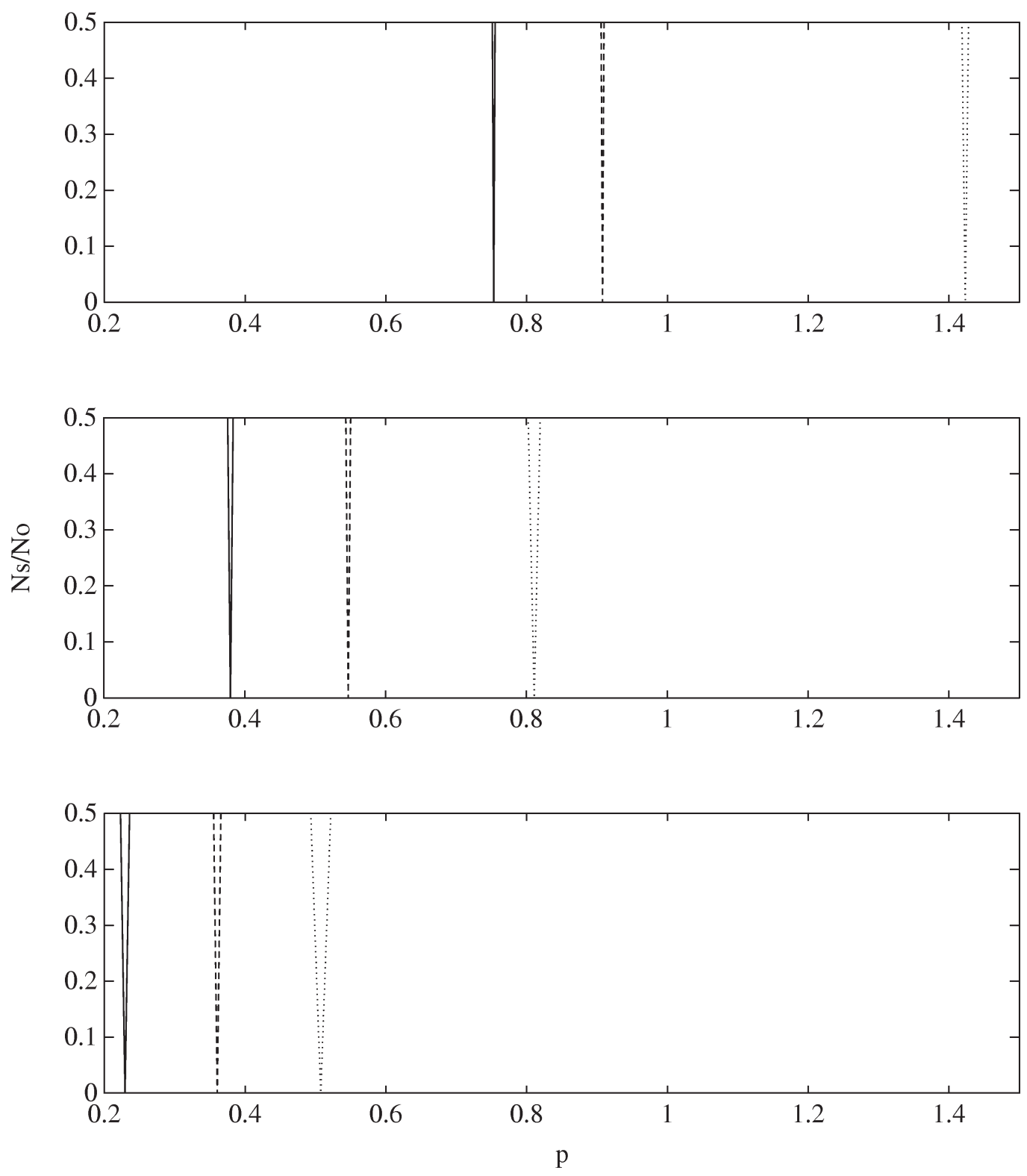

Fig. 5. Unstable regions for a cylindrical shell of length ratio $L / R=3$, tensile loading of $\eta_{\mathrm{o}}=0.5 \eta_{\mathrm{cr}}$ and with axial wave number $m=1$. '_-', SS-SS, ' - - -', C-C, ' . . . ', F-F. Upper diagram $-n=1$. Middle diagram $-n=2$. Lower diagram $-n=3$.

$$
\begin{aligned}
& C_{1}(-\cos \varphi L+\cosh \varphi L) \\
& \quad+C_{2}(-\sin \varphi L+\sinh \varphi L)=0, \\
& C_{1}(\sin \varphi L+\sinh \varphi L) \\
& \quad+C_{2}(-\cos \varphi L+\cosh \varphi L)=0
\end{aligned}
$$

with the frequency equation being

$$
\cos \varphi L \cosh \varphi L=1
$$

\section{Numerical results and discussion}

In order to validate the present results, present results are compared with those of Argento and Scott [3] for a clamped-clamped graphite-epoxy laminated cylindrical shell. The present results for the laminated shell are obtained by making the necessary modifications to the material constitutive equations. The graphical results compared in Fig. 3 show that the present results 

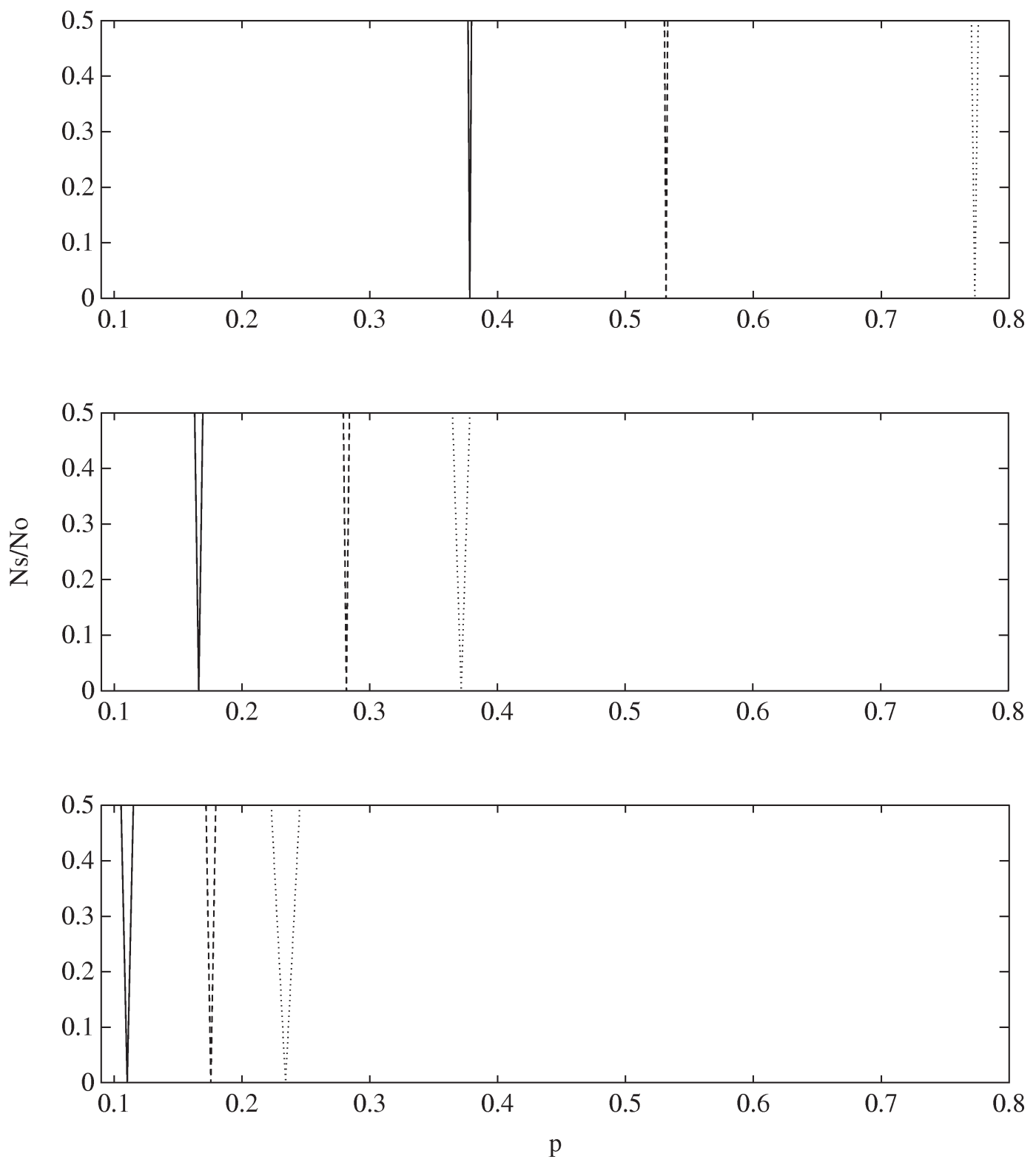

Fig. 6. Unstable regions for a cylindrical shell of length ratio $L / R=5$, tensile loading of $\eta_{\mathrm{o}}=0.5 \eta_{\mathrm{cr}}$ and with axial wave number $m=1$. '_-', SS-SS, ' - -', C-C, ' . . . ', F-F. Upper diagram $-n=1$. Middle diagram $-n=2$. Lower diagram $-n=3$.

are in very good agreement with those of Argento and Scott [3] for the principal instability regions.

The results for the dynamic stability of cylindrical shells of simply-supported-simply-supported (SS-SS), clamped-clamped (C-C) and free-free (F-F) boundary conditions are presented for various length ratios and circumferential wave numbers, $n$. Only the results for the transverse modes are presented as they are usually considered more important with considerably larger unstable regions. The results are for shells of thickness ratio $h / R=0.01$ and for axial wave number $m=1$, meaning that for the F-F case, it is the first non-rigidbody mode in the axial direction.

The nondimensional excitation frequency parameter $p$ is defined as

$$
p=P\left(\frac{\rho R^{2}\left(1-\nu^{2}\right)}{E}\right)^{1 / 2}
$$

The values of $N_{\mathrm{o}}$ are chosen to be in terms of 

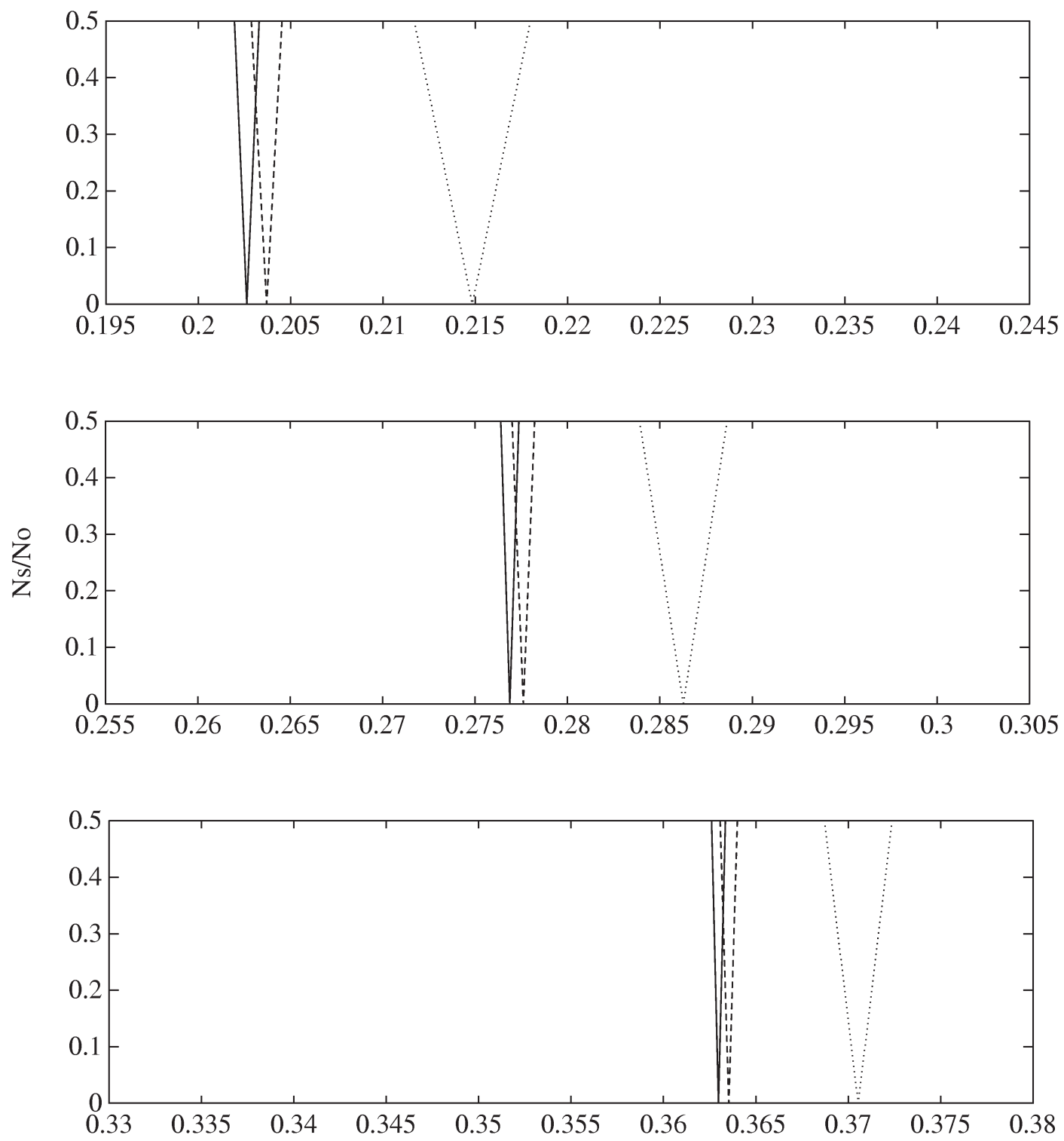

$\mathrm{p}$

Fig. 7. Unstable regions for a cylindrical shell of length ratio $L / R=10$, tensile loading of $\eta_{\mathrm{o}}=0.5 \eta_{\text {cr }}$ and with axial wave number $m=1$. '—', SS-SS, '- - -', C-C, ' $\cdots$. ', F-F. Upper diagram $-n=6$. Middle diagram $-n=7$. Lower diagram $-n=8$.

$P_{\text {cr }}$ which is the critical buckling load of a simplysupported circular cylindrical shell subjected to static compressive axial load. For cylindrical shells of short to intermediate length, as are the cases used here, the buckling load as given by Timoshenko and Gere [15] is

$$
P_{\text {cr }}=\frac{E h^{2}}{\left[3\left(1-\nu^{2}\right)\right]^{1 / 2} R}
$$

and can be nondimensionalized as

$$
\eta_{\mathrm{cr}}=P_{\mathrm{cr}}\left(\frac{1-\nu^{2}}{E h}\right) .
$$

If $\nu$ is taken to be 0.3 ,

$$
\eta_{\mathrm{cr}}=0.5507 \frac{h}{R}
$$

Each unstable region is bounded by two curves originating from a common point from the $p$ axis with $\eta_{\mathrm{s}}=0$. The two curves appear at first glance to be 

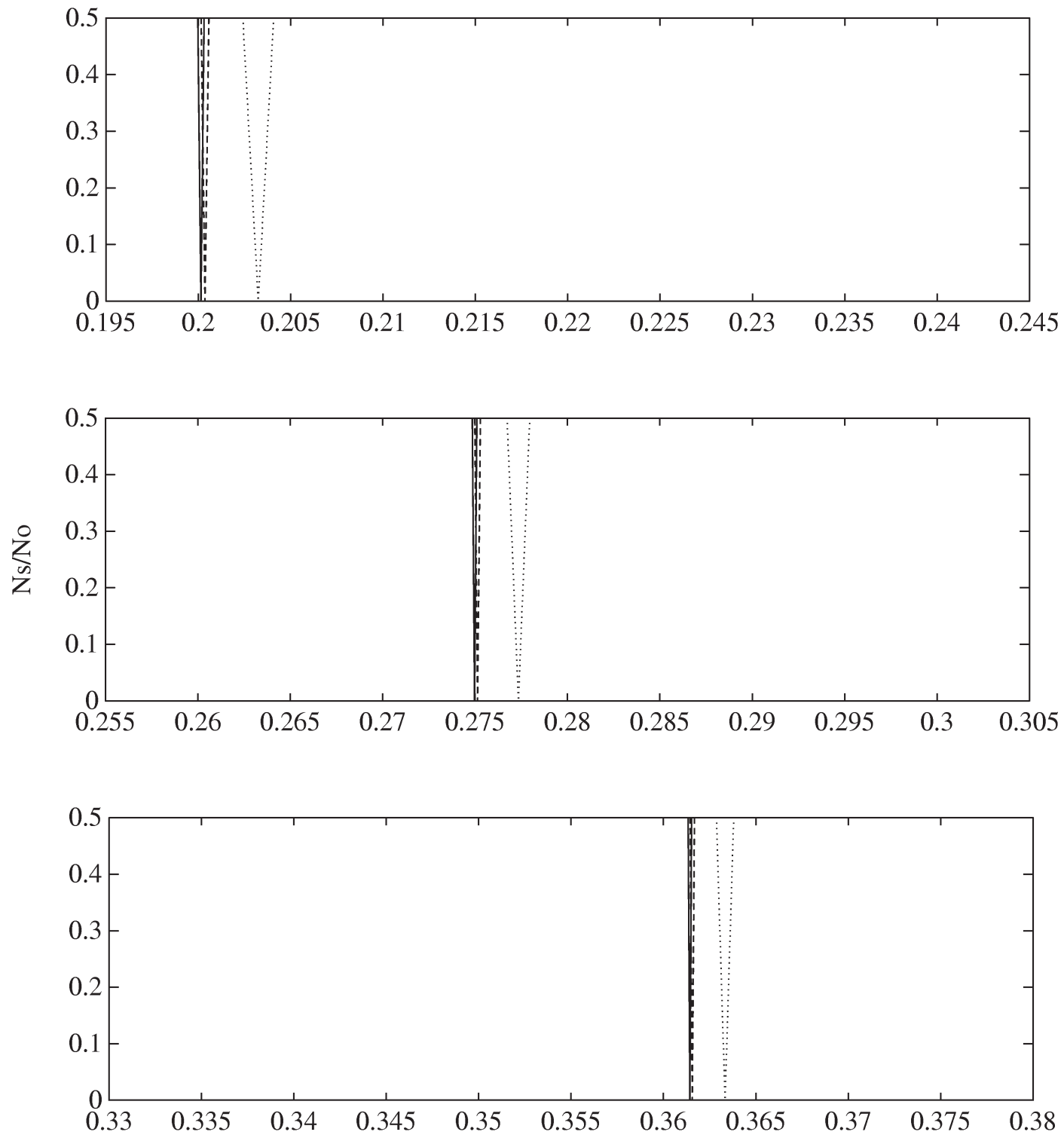

$\mathrm{p}$

Fig. 8. Unstable regions for a cylindrical shell of length ratio $L / R=20$, tensile loading of $\eta_{\mathrm{o}}=0.5 \eta_{\mathrm{cr}}$ and with axial wave number $m=1$. '—', SS-SS, '- - -', C-C, ' $\cdots$. ', F-F. Upper diagram $-n=6$. Middle diagram $-n=7$. Lower diagram $-n=8$.

straight lines but are in fact two very slightly "outward" curving plots. For the sake of tabular presentation, the angle subtended, $\Theta$, is introduced. It is calculated based on the arctangent of the right-angled triangle, $a b c$, obtained by halving the whole unstable region as shown in Fig. 2. This angle gives a good measure of the size of the unstable region as calculations done with the smaller similar triangle, $a b^{\prime} c^{\prime}$ (see Fig. 2), are within $0.2 \%$.

Table 1 and Figs 4 to 6 gives the results for an isotropic cylindrical shell of linear parameters $L / R=$ 1, 3, 5 and circumferential wave numbers $n=1,2,3$ with the axial loading being $\eta_{\mathrm{o}}=0.5 \eta_{\mathrm{cr}}$. Table 2 and Figs 7 to 9 gives the results for an isotropic cylindrical shell of linear parameters $L / R=10,20,30$ and circumferential wave numbers $n=6,7,8$ with the axial loading again being $\eta_{\mathrm{o}}=0.5 \eta_{\mathrm{cr}}$. The choice of these cases are based on previous studies on effects of boundary conditions on the free vibration of cylindrical shells by Lam and Loy [8]. An important aspect of 

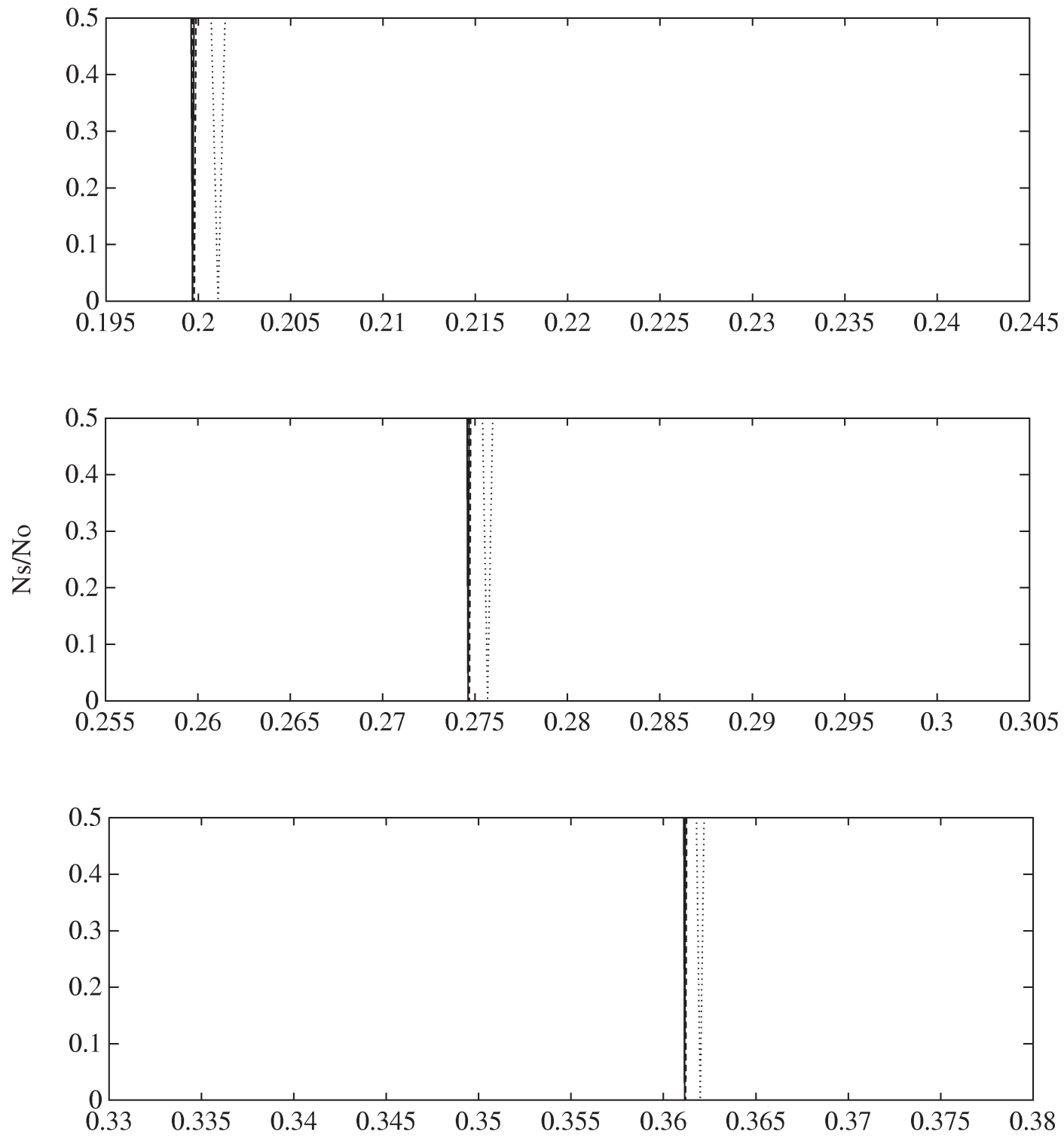

$\mathrm{p}$

Fig. 9. Unstable regions for a cylindrical shell of length ratio $L / R=30$, tensile loading of $\eta_{\mathrm{o}}=0.5 \eta_{\mathrm{cr}}$ and with axial wave number $m=1$. '_-', SS-SS, ' - - ', C-C, ' . . . ', F-F. Upper diagram $-n=6$. Middle diagram $-n=7$. Lower diagram $-n=8$.

the present study is to determine if effects of boundary conditions on the free vibration analysis corresponds to that of dynamic stability analysis.

In the conclusions from Lam and Loy [8] for the effects of boundary conditions on the free vibration of cylindrical shells, it was found that for low $L / R$ and low $n$ values, the natural frequencies were distinct for different boundary conditions. The present study can easily be related to the free vibration study in that the points of origin of the principal unstable regions cor- respond to twice the natural frequencies of the axially loaded shells. The present study confirms the conclusions of the earlier study by Lam and Loy [8] in that the unstable regions are distinct in their points of origin at for different boundary conditions at low $L / R$ and low $n$ values. This can easily be seen from Table 1 and Figs 4 to 6 . Some general observations from the present study are that the sizes of the unstable regions invariably decrease as $L / R$ increase. Also, for the three boundary conditions considered, the F-F case 
always has the highest point of origin and largest unstable region size for any particular mode. The SS-SS case always has the lowest point of origin. As for smallest unstable region size, it can either be the SS-SS case or the $\mathrm{C}-\mathrm{C}$ case.

It was also noted in the study by Lam and Loy [8] that regardless of boundary conditions, all frequencies of similar mode converge with increasing $n$. In that study, the rate of convergence was found to be faster for longer shells. In the present study, the points of origin for different boundary conditions do indeed show convergence behavior as $n$ increases. This can be observed from Table 2 and Figs 7 to 9. The rate of convergence is also found to be faster for longer shells. However, it is important to note here that the sizes of the unstable regions do not exhibit corresponding convergence behavior. This can be observed from Table 2 where is is obvious that the sizes of the unstable regions associated with the different boundary conditions maintain their relative differences at high $n$ values and even for long shells of $L / R=30$. It is also interesting to note here that for long shells, the points of origin are rather insensitive to changes in length but the sizes of the unstable regions are considerably more sensitive to corresponding length changes.

\section{Conclusion}

The dynamic stability of cylindrical shells with three types of boundary conditions (SS-SS, C-C, F-F) has been examined. The F-F case exhibited the highest point of origin and largest unstable region size for any particular mode while the SS-SS case always had the lowest point of origin. The smallest unstable region size is not conclusive and may be associated with the C-C case or the SS-SS case depending on the particular mode. For the different boundary conditions, the points of origins were found to converge at high circumferential wave numbers $n$ with longer shells having higher rates of convergence. However, the sizes of the unstable regions did not display signs of convergence and always maintained their relative differences.

\section{References}

[1] A. Argento, Dynamic stability of composite circular cylindrical shells subjected to combined axial and torsional loading, J. Composite Materials 27 (1993), 1722-1738.

[2] A. Argento and R.A. Scott, Dynamic instability of layered anisotropic circular cylindrical chells, part I: Theoretical development, J. Sound and Vibration 162 (1993), 311-322.

[3] A. Argento and R.A. Scott, Dynamic instability of layered anisotropic circular cylindrical shells, part II: Numerical results, J. Sound and Vibration 162 (1993), 323-332.

[4] C.W. Bert and V. Birman, Parametric instability of thick, orthotropic, circular cylindrical shells, Acta Mechanica $\mathbf{7 1}$ (1988), 61-76.

[5] R.D. Blevins, Formulas for Natural Frequency and Mode Shape, Van Nostrand-Reinhold, New York, 1979.

[6] V.V. Bolotin, The Dynamic Stability of Elastic Systems, Holden-Day, San Francisco, 1964.

[7] L.R. Koval, Effect of longitudinal resonance on the parametric stability of an axially excited cylindrical shell, J. Acoustical Society of America 55 (1974), 91-97.

[8] K.Y. Lam and C.T. Loy, Effects of boundary conditions on frequencies of a multi-layered cylindrical shell, J. Sound and Vibration 188 (1995), 363-384.

[9] C.L. Liao and C.R. Cheng, Dynamic stability of stiffened laminated composite plates and shells subjected to in-plane pulsating forces, J. Sound and Vibration 174 (1994), 335-351.

[10] C.L. Liao and C.R. Cheng, Dynamic stability of stiffened laminated composite plates and shells subjected to in-plane pulsating forces, Int. J. Numerical Methods in Engineering 37 (1994), 4167-4183.

[11] C. Massalas, A. Dalamangas and G. Tzivanidis, Dynamic instability of truncated conical shells, with variable modulus of elasticity, under periodic compressive forces, J. Sound and Vibration 79 (1981), 519-528.

[12] K. Nagai and N. Yamaki, Dynamic stability of circular cylindrical shells under periodic compressive forces, J. Sound and Vibration 58 (1978), 425-441.

[13] H.R. Radwan and J. Genin, Dynamic instability in cylindrical shells, J. Sound and Vibration 56 (1978), 373-382.

[14] K. Shirakawa, Dynamic stability of cylindrical shells taking into account in-plane inertia and in-plane disturbance, Bulletin of the JSME 23 (1980), 163-169.

[15] S.P. Timoshenko and J.M. Gere, Theory of Elastic Stability, 2nd edn, McGraw-Hill, New York, 1961.

[16] J.C. Yao, Dynamic stability of cylindrical shells under static and periodic axial and radial loads, AIAA Journal 1 (1963), 1391-1396. 

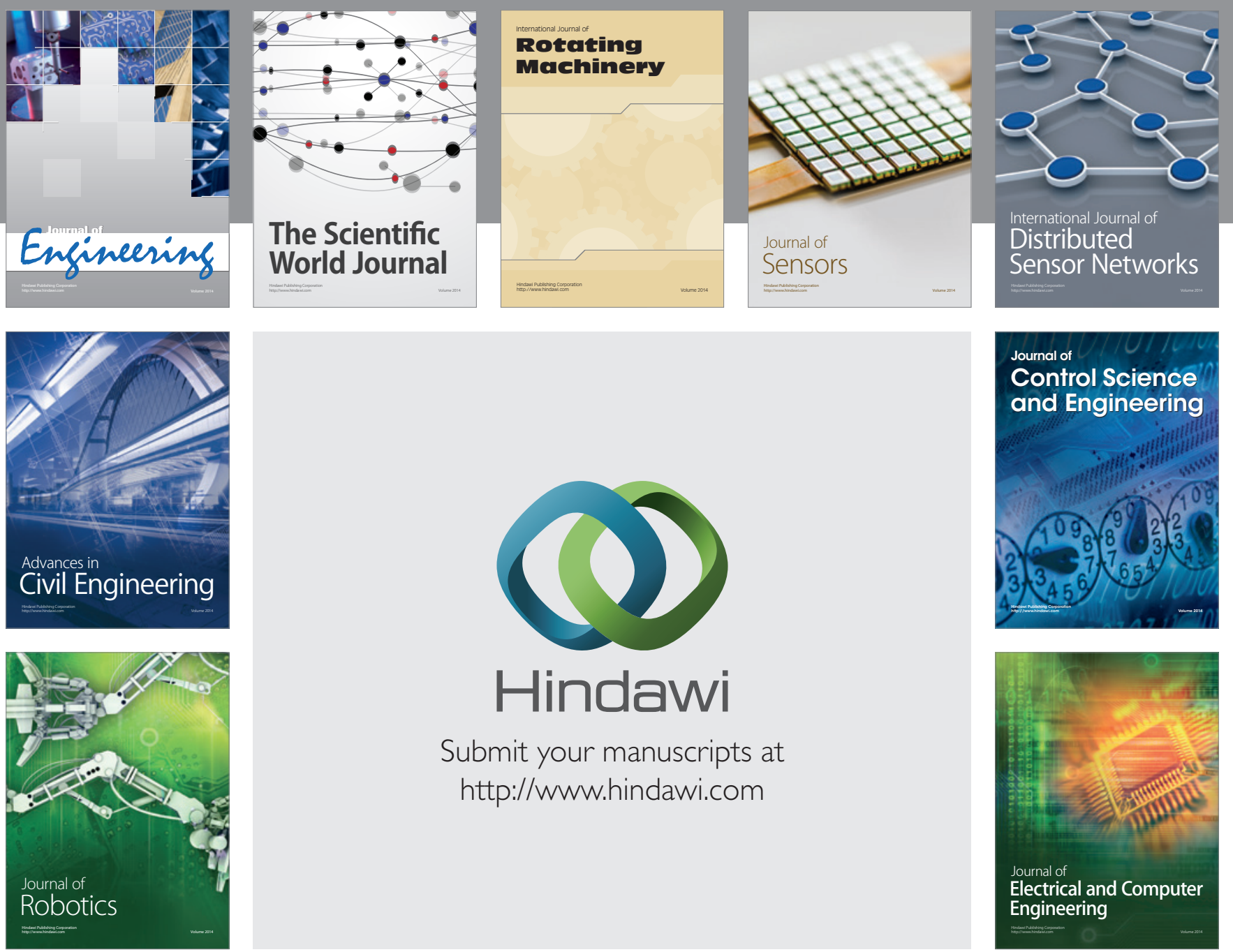

Submit your manuscripts at

http://www.hindawi.com
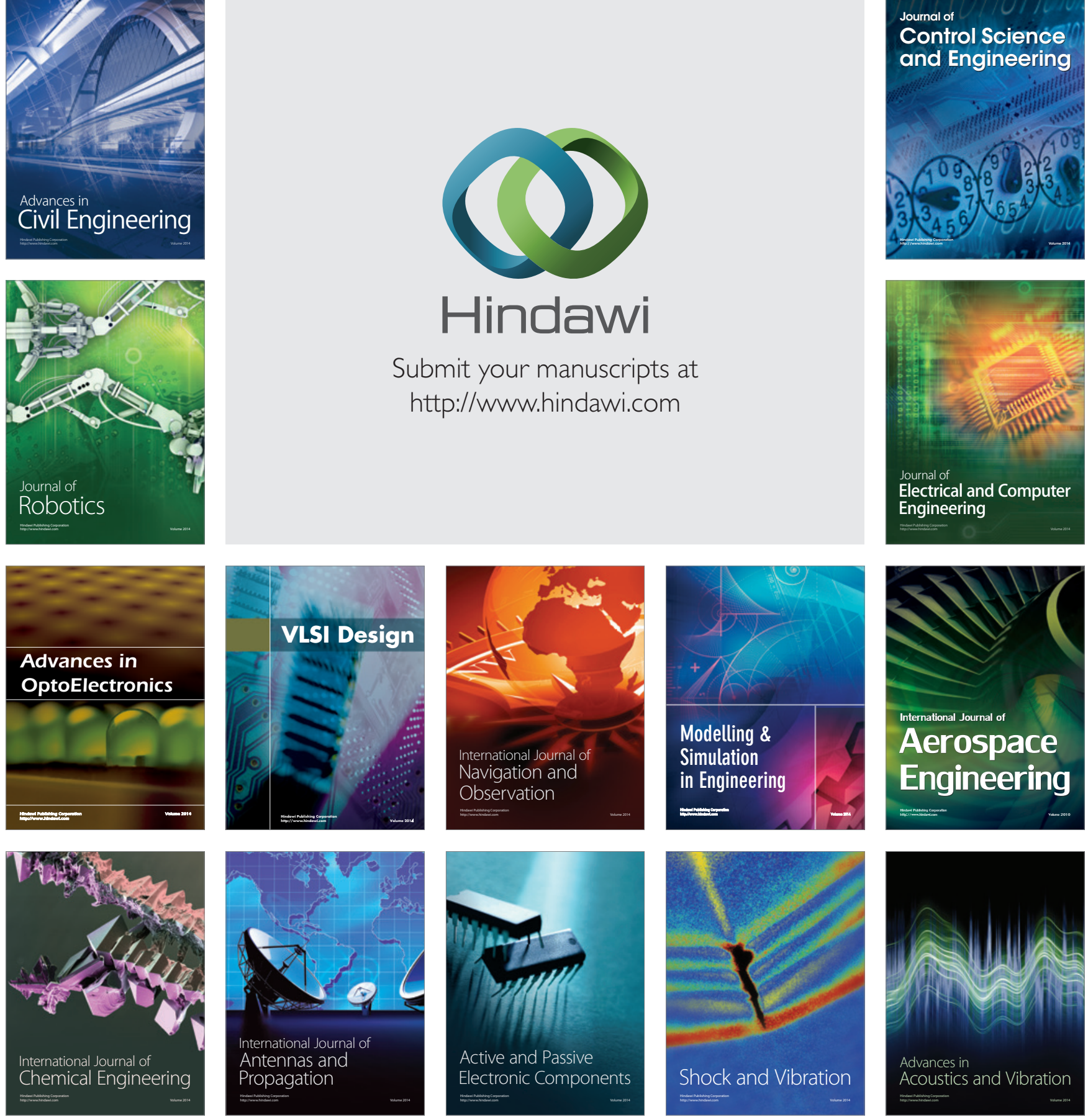